\title{
Levator Labii Superioris
}

National Cancer Institute

\section{Source}

National Cancer Institute. Levator Labii Superioris. NCI Thesaurus. Code C53164.

A thin, quadrilateral muscle originating at the lower margin of the orbit and attaching to

the muscles of the upper lip to elevate the lip. 